\title{
Rechargeable Automated Implantable Cardioverter Defibrillator
}

\author{
Dr.Vasuki. ${ }^{1}$, Aarthika. L ${ }^{2}$, Abhinaya. $\mathrm{R}^{3}$, Lekshmi Bhadran ${ }^{4}$ \\ ${ }^{1}$ Bio - medical Engineering, Bharath University, India. \\ ${ }^{2}$ Bio- medical Engineering, Bharath University, India. \\ ${ }^{3}$ Bio - medical Engineering, Bharath University, India. \\ ${ }^{4}$ Bio - medical Engineering, Bharath University, India.
}

\begin{abstract}
An automated Implantable Cardioverter Defibrillator (AICD) is a small battery powered electrical impulse generator, implanted in patients who are in the risk of sudden cardiac death or ventricular tachycardia, and is programmed to detect the cardiac arrhythmia. Components of AICD are Leads, Electrode and Generator ${ }^{[1]}$. The electric shock is created by the generator carried by leads and delivered with the electrodes. The generator is of the size of 2 inches approximately, and 3 ounces weight. Working of AICD includes rectification of arrhythmia by means of pacing Cardioversion defibrillation. Current devices have appreciable functions including diagnosis, stored electro-grams and verification of shock appropriations and battery longevity upto 6 yrs. Here using mutual inductance ${ }^{[2]}$ functions carried out with the help of a transcutaneous transformer and storing electric charge through magnetic field absolute longevity of the battery could be reached. Since, battery longevity has been tremendously increased the recurrence of surgery can be prevented. Thus, our device will bring remarkable revolution in cardiology by saving human lives from arrhythmia and periodical replacement of the device.
\end{abstract}

Keywords:-ADC, Faraday's Law, Micro-controller (MC), Mutual inductance, Transcutaneous transformer.

\section{Introduction:-}

Various implantable devices are battery powered and this battery has been a matter of concern as they may not last for longer duration and thus requires frequent replacements. Various methods to recharge battery ${ }^{[3]}$ inside the patient's body using bio-thermal, bio-mechanical, and RF methods have been proposed and were seen not producing enough charge to power the device and may further require supplementary circuits as a reservoir. Recharging technique using transcutaneous transformer is proposed through this paper. Taking this as an initial step various implantable medical devices can be powered continuously without any need for replacement.

Mutual inductance property is used to recharge the AICD setup. It involves two coils (transcutaneous transformer ${ }^{[4],[5]}$ ) primary and secondary coils separated with skin in between them. The energy transmission from the primary to the secondary coil is facilitated using magnetic field produced in the primary coil that induces current in the secondary coil according to the Faraday's Law of induction. A micro-controller unit is used to control and monitor the flow of charge and the battery's status is displayed using an LED. An ADC is used to enhance communication between the MC and the peripheral's of the setup.

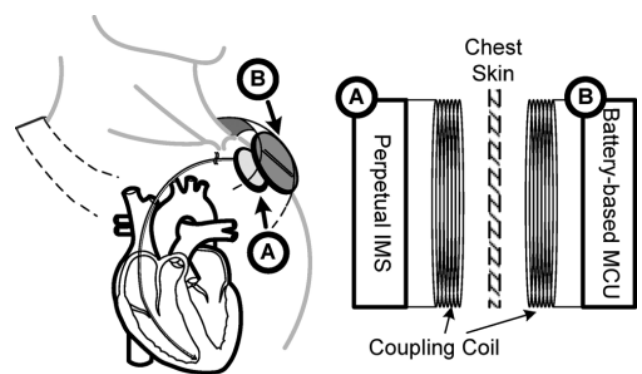

Fig 1.1 Transcutaneous Transformer. 


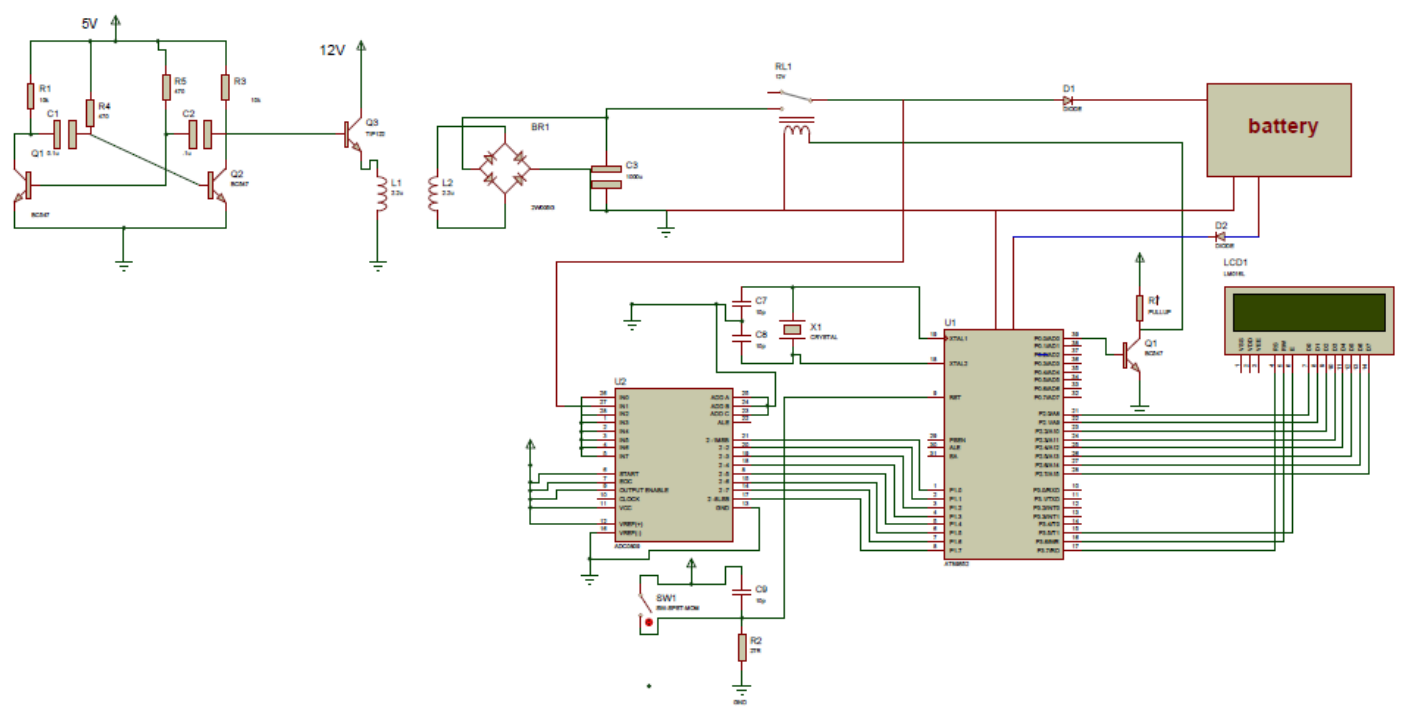

Fig 1.2 Recharging circuit of AICD.

\section{Conclusion:-}

Considerable amount of charge can be produced and stored using this technique and thus repeated surgery and trauma to the patient is avoided. Most complications are minor and easily treated, such as pain, bleeding and bruising at the implant site. An infrequent complication is pneumothorax, a condition when the lung is accidentally punctured during the insertion of the lead. Air then leaks into the chest cavity, causing the lung to collapse. The patient is subjected to regular periodic recharging battery ${ }^{[6][7]}$ of the device to keep up with its proper functioning and reliable outcome. The method can be used to recharge various other implantable assisting devices as well. Further application of sophisticated and technically enhanced battery can be applied for better performance and longitivity.

\section{References:-}

[1] Technical Information VNS TheraphyTM DemiPulseTM Model 103 Generator and VNS TheraphyTM DemiPulseTM Model 104 Generator For Healthcare Professionals. Cyberonics, Apr. 2008

[2] S. B. Lee, H.M. Lee, M. Kiani, U.M. Jow, and M. Ghovanloo, An inductively powered scalable 32-channel wireless neural recording system-on-a-chip for neuroscience applications, in Solid-State Circuits Conference Digest of Technical Papers (ISSCC), 2010 IEEE International, Feb. 2010, $120-121$.

[3] (2009) XcellionTM rechargeable batteries. [Online]. Available:http://greatbatchmedical.com/assets/products/XcellionBatteries.pdf

[4] H. Lim, Y. Yoon, C. Lee, I. Park, B. Song, and J. Cho, Implementation of a transcutaneous charger for fully implantable middle ear hearing device, Jan. 2005, $6813-6816$.

[5] Z. Tang, R. Sclabassi, C. Sun, S. Hackworth, J. Zhao, X. Cui, and M. Sun, Transcutaneous Battery Recharging By Volume Conduction and its Circuit Modeling, in Engineering in Medicine and Biology Society, 2006. EMB S'06, 28th Annual International Conference of the IEEE, Sept. 2006, $644-647$.

[6] C.-C. Huang, S.-F. Yen, and C.-C. Wang, A Li-ion Battery Charging Design for Biomedical Implants, in Proc. IEEE Asia Pacific Conference on Circuits and Systems, (APCCAS'08), Dec. 2008, 400 -403.

[7] C.-W. Chuang, E. Go Chua, Y.-S. Lai, and W.-C. Fang, RF-powered Li-ion Battery Charger for Biomedical Applications, Apr. $2009,187-189$ 